\title{
An Intelligent Watchdog Model for Quality Control of an Affective Bio-Greening Material
}

\author{
Mirwan Ushada and Haruhiko Murase \\ Graduate School of Life and Environmental Sciences, Osaka Prefecture University, \\ 1-1 Gakuen-cho, Naka-ku, Sakai, Osaka 599-8531, Japan
}

(Received February 6, 2009)

\begin{abstract}
This paper highlighted a new methodology for quality control of an Affective Bio-greening Material (ABGM). ABGM is a bio-greening material which is produced in non-uniform controlled environment to generate the dynamic appearance for satisfying the different consumer. In this methodology, the consumer evaluated easily the material affectivity using its appearance in the questionnaires. Subsequently, this appearance was detected in production using textural features extracted from grey level co-occurrences texture analysis and water content. Hence, quality control was defined as the function of irreversible change of textural appearance which was stimulated by water content. Artificial Neural Network (ANN) was used to develop an intelligent watchdog model. The model was defined that material affectivity can be controlled from textural images and water content in a given local temperature and relative humidity. The model was demonstrated via a case study of Sunagoke moss greening (Rhacomitrium japonicum). The trained ANN model generated satisfied correlation between measured and predicted values and minimum inspection error. The research results concluded the possibility to control the affectivity of a bio-greening material in a non-uniform environment based plant factory production using an intelligent watchdog model.
\end{abstract}

Keywords : affective sunagoke moss, artificial neural network, non-uniform controlled environment, textural features, water content

\section{INTRODUCTION}

Material affectivity is one of the most important factors to satisfy the consumer. Nagamachi (1995) emphasized the significance of incorporating affectivity to the consumer into a material quality. Affectivity is defined as the visual satisfaction of consumer when they see a material due to its appearance (Yang et al., 1999).

In this paper, we want to highlight an Affective Bio-greening Material (ABGM). ABGM is a bio-greening material which is produced in non-uniform controlled environment to generate the dynamic appearance for satisfying the different consumer. Takahashi et al. (2002) have suggested the importance of controlling the non-uniform environment in a plant factory.

Quality control is highly required for production of ABGM. There is a keen demand for a watchdog model to control the quality (Hori et al., 2002). The recent researches of watchdog model are focusing on bio-material parameters (Zaidi et al., 1999; Murase and Ushada, 2006; Ushada et al., 2007) and none of them have incorporated the material affectivity.

Corresponding author: Mirwan Ushada, fax:+81-72-254-9918, e-mail : mirwan@bics.envi.osakafu-u.ac.jp 
Therefore, a new concept for quality control was developed using an intelligent watchdog model as shown in Fig. 1. We defined the quality of ABGM as the affectivity status of material appearance to satisfy the consumer. Quality control is defined as the function of the irreversible change of textural appearance which was stimulated by water content in the non-uniform environment. Hence, the watchdog model is defined that material affectivity could be controlled from textural images and water content in a given non-uniform controlled environment.

Fusing various parameters of bio-material, controlled environment and consumer information requires a robust modeling tool. Artificial Neural Network (ANN) is required for developing an intelligent watchdog model. It is due to its capability to model large number of non linear parameters in biosystems (Murase et al., 1997; Reed and Marks II, 1999).

The objectives of this paper were: 1) To develop an intelligent watchdog model for quality control of an affective bio-greening material; 2) To model the relationship between non-uniform controlled environment and affectivity of a bio-greening material by using an ANN model. The model is demonstrated via a case study of Sunagoke moss (Rhacomitrium japonicum).

\section{MATERIALS AND METHODS}

\section{Methodology}

Figure 2 presents a methodology for building an intelligent watchdog model for the quality control.

As the first step, we offered a set of reference sample to the consumer candidates using ques-

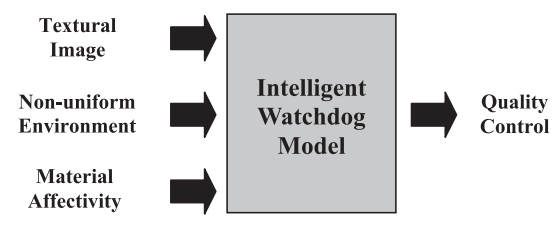

Fig. 1 A new concept of quality control for production of affective bio-greening material.

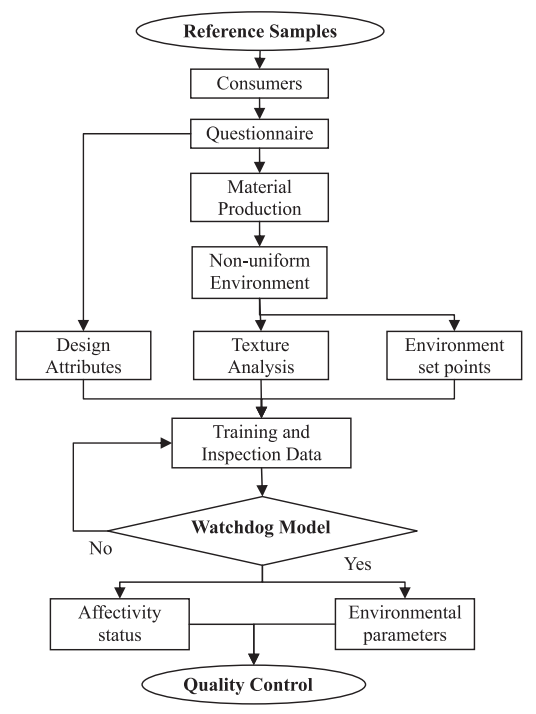

Fig. 2 Methodology for building an intelligent watchdog model. 
tionnaires. These references were produced in a preliminary experiment to provide the textural feature and water status data base in moss production (Ushada et al., 2007). The moss affectivity to the consumer was identified by its design attributes. Based on these attributes, the material was produced in an experiment. Affectivity status was classified using the data base. The hypothesis in moss production is that the affectivity is possible to be detected by its textural image and water content. Hence, the experiment was developed in a non-uniform environment. As the final step, the experiment and questionnaire provided the training and inspection data for an ANN model. The proposed ANN model is expected as an intelligent watchdog model.

\section{Questionnaires}

A total of four reference samples were offered to consumer candidates using 5 point-based Likert scale questionnaire. A total of 102 consumer candidates determined the affectivity of samples by rating the importance of its design attribute. Wet and semi-dry appearances were indicated most preferred as affective moss. Questionnaire concluded the references for affective sample as shown in Figs 3a and 3b.

Besides, questionnaire concluded reference for non-affective samples (Figs $4 a$ and $4 b$ ).

The questionnaire provided illustration that moss samples are applied for the greening technology (Fig. 5a). This technology consists of a set of moss module $(50 \mathrm{~cm} \times 50 \mathrm{~cm})$ (Fig. 5b). The sample $(8 \mathrm{~cm} \times 11 \mathrm{~cm})$ was used as the representation of the module (Fig. $5 \mathrm{c}$ ).

The questionnaire concluded three attributes which contribute significantly to moss affectivity. These attributes were summarized in Table 1. Waterproofing contributes to the structure strength in greening technology. Easy maintenance indicates that consumer preferred moss which does not grow rapidly. Comfortable contribute to the consumer feeling when they used moss as a biogreening material.

\section{Material affectivity to the consumer}

Material affectivity to the consumer was measured by Weighted Average Importance Index (WAII) of a design attribute. Each importance was quantified using weighted average importance index as shown in Eq. (1):

$$
W A I I=\frac{\sum_{k}\left[\left(N_{A} \times k\right)\right]}{N_{t}}
$$

where $N_{A}$ is number of respondents at importance value $k, N_{t}$ is total number of respondents, and $k$ is grade of importance (1, 2, 3, 4 and 5 of Likert response).

\section{Non-uniform environment}

Based on three design attributes, nine new samples of moss were produced. These samples were grown in two growth chambers (Biotron, NK 50, Osaka, Japan). Several positions inside the chamber were used to simulate non-uniform environmental condition. The objective was to analyze the effect of different local environment to the moss affectivity.

Both chambers were equipped with the controller of temperature and relative humidity. In order to simulate a natural diurnal cycle, the lamps were active for $12 \mathrm{~h}$ from $09.00 \mathrm{a} . \mathrm{m}$. until 09.00 p.m. and automatically turned off every night. The initial set points of temperature $T_{\text {setpoint }}$ and relative humidity $R H_{\text {setpoint }}$ were set using controller of the chamber in a range of 15.4 to $21.4^{\circ} \mathrm{C}$ and 73 to $93.7 \%$ respectively. Subsequently, the non-uniform environment was set. Figure 6 indicated an

Table 1 Design attributes for affective moss.

\begin{tabular}{cc}
\hline Attributes & Definition \\
\hline Waterproofing & Structure of greening technology \\
Easy maintenance & Controlled growth \\
Comfortable & Feeling from appearance \\
\hline
\end{tabular}




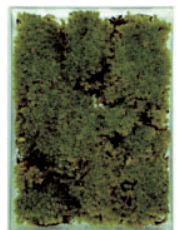

(a)

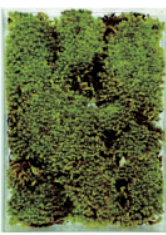

(b)

Fig. 3 Reference appearances for affective moss: (a) Wet moss; (b) Semi-dry moss.

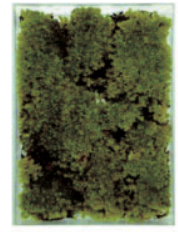

(a)

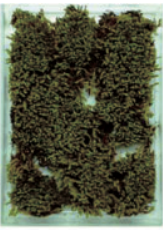

(b)

Fig. 4 Reference appearances for non-affective moss: (a) Soak moss; (b) Dry moss.

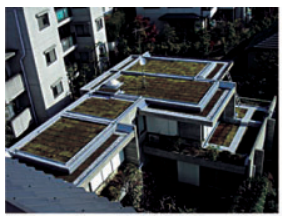

(a)

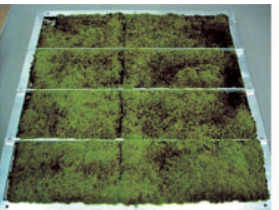

(b)

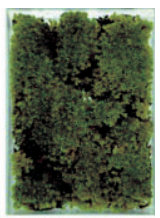

(c)

Fig. 5 Material structure for moss production: (a) Greening application; (b) Module; (c) Sample.

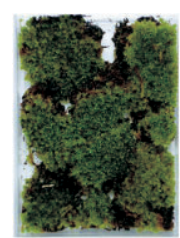

(a)

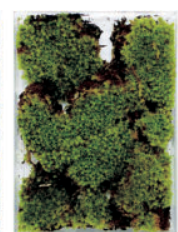

(b)

Fig. 8 The developed affective appearances which have similar textural feature values with reference samples (Figs. 3a and 3b): (a) Wet moss (Day 4); (b) Semi-dry moss (Day 9).

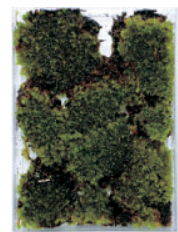

(a)

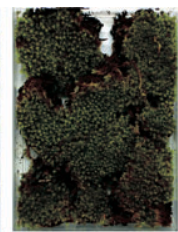

(b)

Fig. 9 The developed non-affective appearances for quality control which have similar textural feature values with reference samples (Figs. 4a and 4b): (a) Soak moss (Day 1); (b) Dry moss (Day 10). 


\section{AN INTELLIGENT WATCHDOG MODEL}

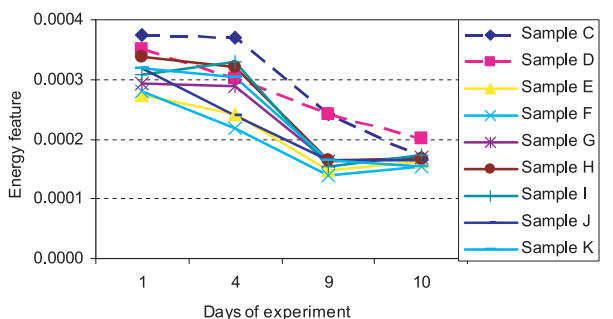

(a)

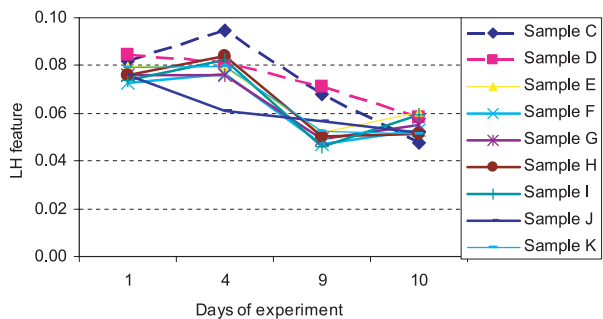

(b)

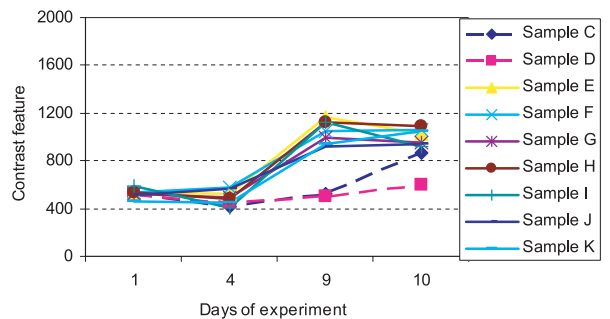

(c)

Fig. 10 Dynamic textural features for detecting the moss affectivity: (a) Energy; (b) LH; (c) Contrast.

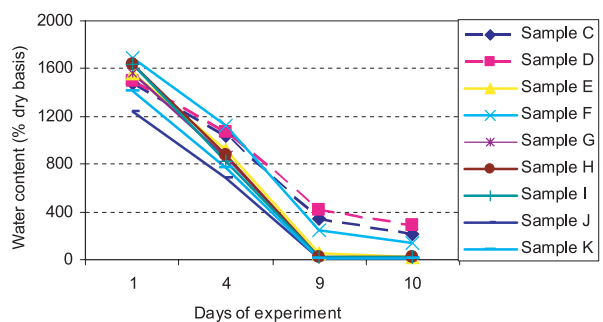

(a)

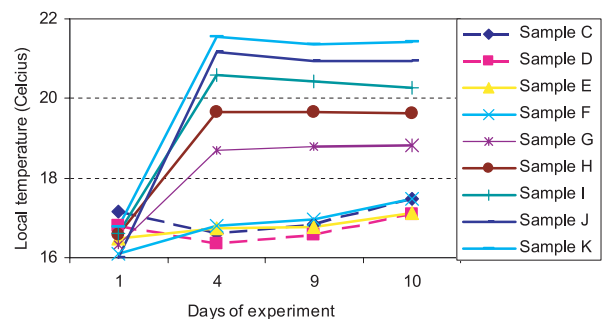

(b)

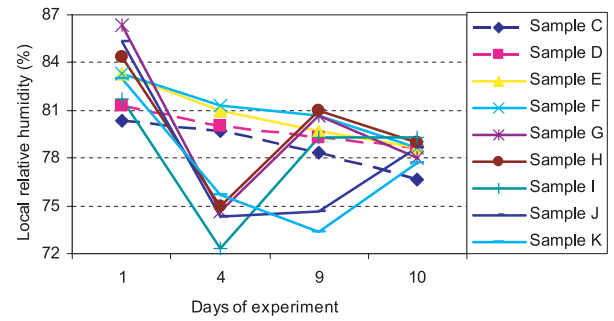

(c)

Fig. 11 Dynamic water content and controlled environment for detecting the moss affectivity: (a) Water content; (b) Local temperature $T_{\text {local }}$; (c) Local relative humidity $R H_{\text {local }}$. 


\section{USHADA AND H. MURASE}

example of non-uniform local temperature $T_{\text {local }}$ and relative humidity $R H_{\text {local }}$ distribution inside a chamber.

$T_{\text {local }}$ and $R H_{\text {local }}$ from each position of sample were measured (Thermo-recorder TR-72U, T\&D Cooperation, Osaka, Japan). Table 2 summarized the average local $T_{\text {local }}$ and $R H_{\text {local }}$ for each sample position during the experiment.

Table 2 Average local environmental status for each sample position during the experiment.

\begin{tabular}{ccc}
\hline Samples & $T_{\text {local }}\left({ }^{\circ} \mathrm{C}\right)$ & $R H_{\text {local }}(\%)$ \\
\hline C & $17.21 \pm 0.53$ & $78.37 \pm 2.87$ \\
D & $16.90 \pm 0.46$ & $79.33 \pm 2.61$ \\
E & $16.94 \pm 0.36$ & $79.41 \pm 2.28$ \\
F & $17.12 \pm 0.39$ & $79.22 \pm 2.77$ \\
G & $18.78 \pm 0.82$ & $78.57 \pm 3.26$ \\
H & $19.70 \pm 0.96$ & $76.73 \pm 4.05$ \\
I & $20.31 \pm 1.05$ & $76.31 \pm 4.29$ \\
J & $20.68 \pm 1.26$ & $75.86 \pm 5.53$ \\
K & $21.07 \pm 1.15$ & $75.35 \pm 4.45$
\end{tabular}

Table 3 Affectivity status classification.

\begin{tabular}{ccccc}
\hline Quality status & Energy $\left(\times 10^{-4}\right)$ & LH $\left(\times 10^{-1}\right)$ & Contrast & Affectivity status \\
\hline Affective Wet & $3 \leq X<5.5$ & $0.72 \leq X<1.13$ & $279<X \leq 514$ & 1 \\
Affective Semi-Dry & $2 \leq X<3$ & $0.61 \leq X<0.72$ & $514<X \leq 723$ & 0.5 \\
Non-affective Soak & $X \geq 5.5$ & $X \geq 1.13$ & $X \leq 279$ & 0 \\
Non-affective Dry & $X<2$ & $X<0.61$ & $X>723$ & 0 \\
\hline
\end{tabular}

\begin{tabular}{|c|c|}
\hline $15^{\circ} \mathrm{C}{ }^{80 \%}$ & $17^{\circ} \mathrm{C}$. $75 \%$ \\
\hline $19^{\circ} \mathrm{C}$. $70 \%$ & $19^{\circ} \mathrm{C}$ \\
\hline $17^{\circ} \mathrm{C}$. $72 \%$ & $81 \%$ \\
\hline $16^{\circ} \mathrm{C}=75 \%$ & $15^{\circ} \mathrm{C}$ \\
\hline
\end{tabular}

Fig. 6 An example of non-uniform temperature and relative humidity distribution inside a chamber.

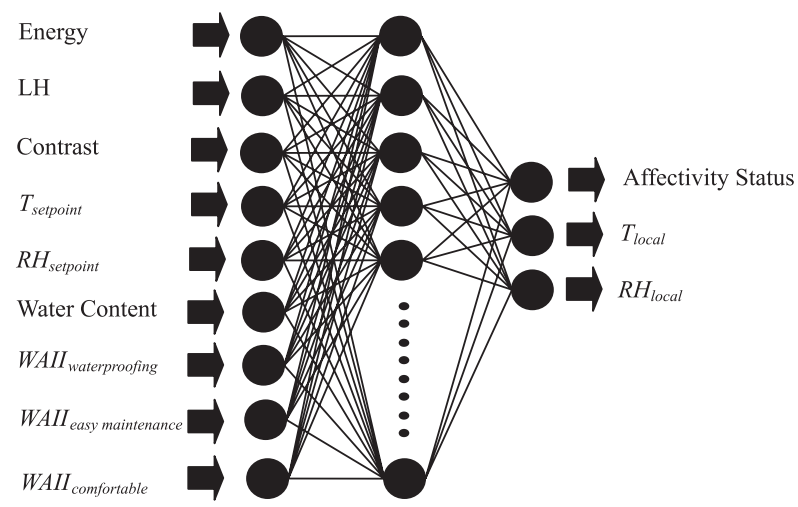

Fig. 7 Artificial neural network for an intelligent watchdog model. 


\section{AN INTELLIGENT WATCHDOG MODEL}

\section{Image and data acquisition}

Samples were left to dry gradually for 17 days to generate the irreversible change of textural appearance. Throughout the drying periods, all samples were weighed and removed safely for textural image acquisition. The moss was placed in plastic case anti static in order to define area of interest in image acquisition. The image and data acquisition consisted of Charged Couple Device (CCD) camera Sony DSW-1, camera tripod SLIK 8006-111 (38.5 cm from object), lighting lamp condition NEC $15 \mathrm{~W}$ ( $38.5 \mathrm{~cm}$ from object), illuminating base plate $38.5 \times 27 \mathrm{~cm}^{2}$ and plastic case with area of interest $8 \times 11 \mathrm{~cm}^{2}$. At the same time, water content was measured based on dry basis (\% dry basis) using electronic balance weight.

6. Texture analysis

The texture analysis can be considered as one of applicable techniques for extracting textural features of image and application for appearance pattern recognition. The co-occurrence matrix or known as grey tone spatial dependence matrix is a second order image variation. It can provide a basis for a number of textural features (Haralick et al., 1973; Murase et al., 1997).

Equations (2), (3) and (4) summarized the textural features which are the most critical to plant growth indices (Murase et al., 1997):

(a) Energy:

$$
\sum_{i=1}^{n} \sum_{j=1}^{n} P_{(1,0)}(i, j)^{2}
$$

(b) Contrast:

$$
\sum_{i=1}^{n} \sum_{j=1}^{n}(i-j)^{2} P_{(1,0)}(i, j)
$$

(c) Local Homogeneity (LH):

$$
\sum_{i=1}^{n} \sum_{j=1}^{n}\left\{P_{(1,0)}(i, j)\right\} /\left\{1+(i-j)^{2}\right\}
$$

where :

$\mathrm{d}=$ distance between two neighboring resolution cells

$\mathrm{q}=$ angle between two neighboring cells

$P_{(1,0)}(i, j)=$ joint probability density function at $d=1$ and $q=0$

$i, j=$ notation for grey tone

In this study, the texture analysis source code program was built using Microsoft Visual C++ 6.0 based on grey level co-occurrence matrix (MI, 1998). The program consisted of four modules covering co-occurrence matrix, energy, LH and contrast features extraction. The co-occurrence matrix was calculated based on normalization value. Pre-processing methods were done using Image-J and Pop Imaging software (NIH, 2004; DBK, 2004). It consists of grey level value extraction from textural image and also determination of area of interest.

\section{Affectivity status}

Affectivity status was classified using the data base as shown in Table 3 (Ushada et al., 2007). Affective wet was indicated using scale 1 , affective semi-dry using scale 0.5 and both non-affective soak and dry using scale 0 . The affectivity status is indexed into the value between 1 and 0 to fit the sigmoid function of the proposed ANN.

\section{Intelligent watchdog model}

An intelligent watchdog model is defined as a black box relationship between non-uniform controlled environment and affectivity of a bio-greening material. Its function for quality control can be defined as dynamic variation of moss textural image, water content, $T_{\text {local }}, R H_{\text {local }}$ and material affectivity. Thus, these relationships were modeled using a three layered ANN as shown in Fig.7.

The inputs were three textural features, $T_{\text {setpoint }}$ and $R H_{\text {setpoint }}$, water content and WAII of three design attributes. The outputs were affectivity status, $T_{\text {local }}$ and $R H_{\text {local. }}$. Affectivity status is expected as threshold quality status for the quality control. Local temperature and relative humidity is 


\section{USHADA AND H. MURASE}

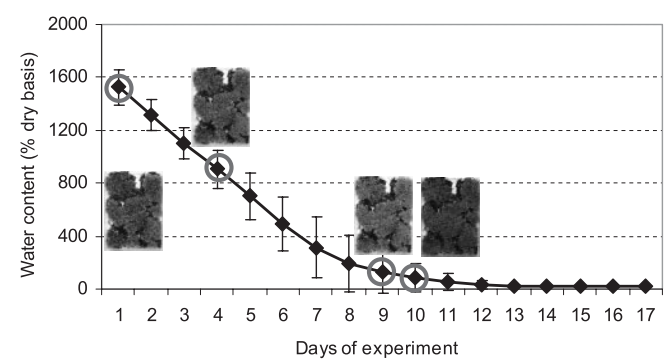

Fig. 12 Average water content profile for controlling the moss affectivity.

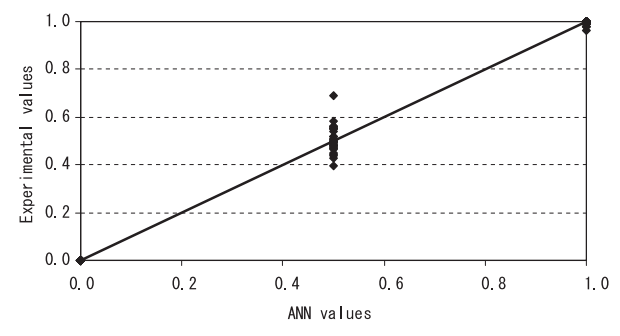

(a)

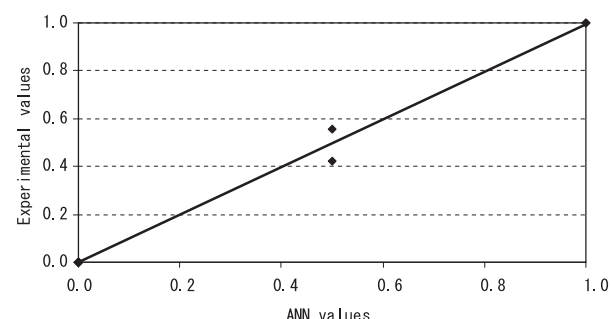

(b)

Fig. 13 Affectivity status comparison between experimental and ANN values: (a) Training; (b) Inspection data.

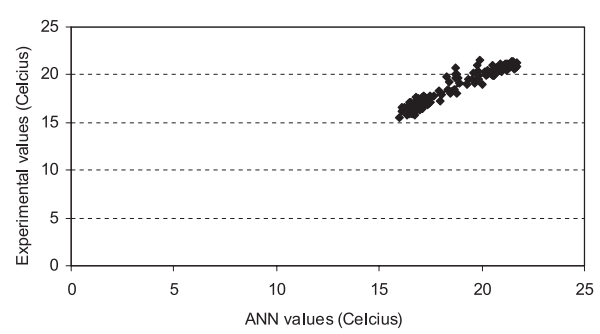

(a)

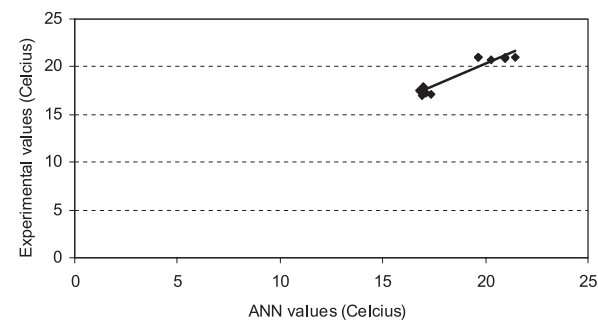

(b)

Fig. 14 Local temperature $T_{\text {local }}$ comparison between experimental and ANN values: (a) Training; (b) Inspection data.

expected as the feedbacks for non-uniform environmental control system in a plant factory.

\section{RESULTS}

Figures $8 \mathrm{a}$ and $8 \mathrm{~b}$ indicated the affective moss which was produced in the non-uniform environment. These moss have the similar value of energy, LH and contrast features with the reference samples (Figs. 3a and 3b) in the different appearance. Therefore, textural features are possible as indicator to generate the various appearances in ABGM production to satisfy the different consumer.

Figures $9 \mathrm{a}$ and $9 \mathrm{~b}$ indicated threshold appearances as the indicator for quality control.

These appearances must be analyzed with the comparison of textural feature patterns. The 
AN INTELLIGENT WATCHDOG MODEL

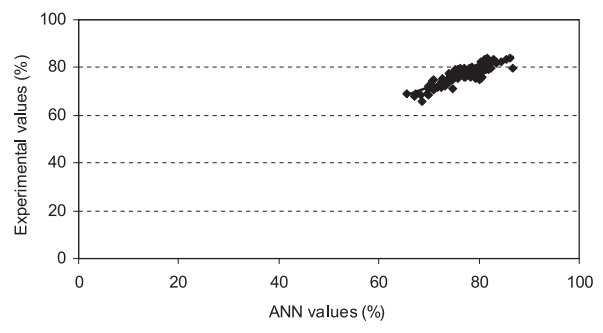

(a)

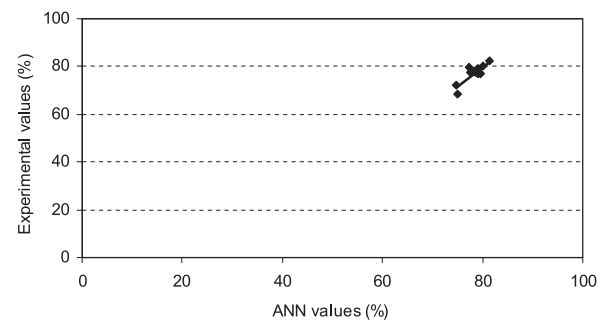

(b)

Fig. 15 Local relative humidity $R H_{\text {local }}$ comparison between experimental and ANN values: (a) Training; (b) Inspection data.

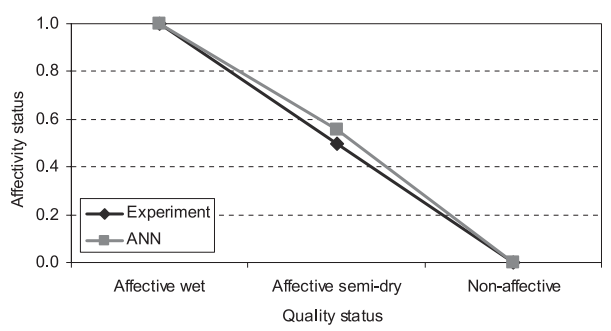

(a)

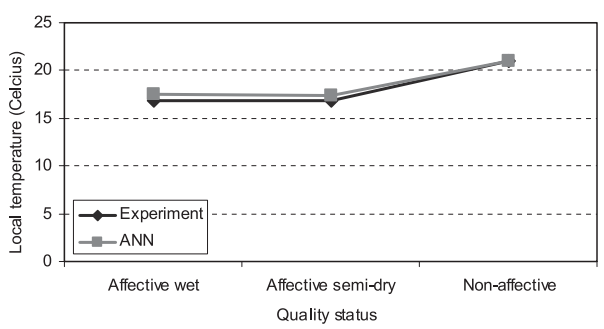

(b)

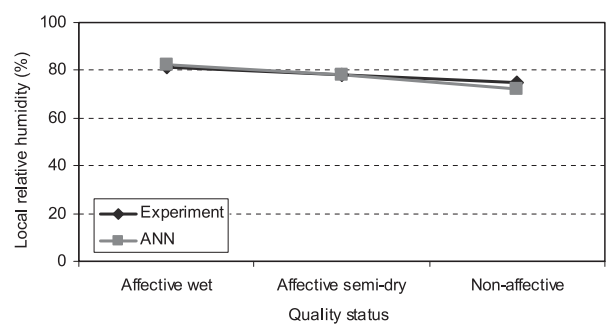

(c)

Fig. 16 Inspection data for different quality status; (a) Affectivity status; (b) $T_{\text {local }}$; (c) $R H_{\text {local }}$.

comparison was made between days 1, 4, 9 and 10 as indicated in Fig. 10. On day 10, all the samples have reached the dry status. Figure 10a indicated decreasing trend of energy feature value. The same trend is indicated in Fig. 10b for LH feature value. Contrast feature has shown the opposite trend in Fig. 10c. In order to clarify these textural features, profiles of water content, $T_{\text {local }}$ and $R H_{\text {local }}$ were observed in Figs. 11a, $11 \mathrm{~b}$ and $11 \mathrm{c}$ respectively. It indicated that there were obvious differences among these days. The comparison showed that there were two threshold points on days of 1 and 10 for preventing affectivity degradation. The day 4 is a time indicator to harvest the wet and day 9 for the semi-dry moss.

\section{DISCUSSION}

\section{Detecting moss affectivity in production}

Figure 10 indicated that samples ' $\mathrm{C}$ ' and ' $\mathrm{D}$ ' have lower values for energy and $\mathrm{LH}$ features 
and higher value for contrast rather than other samples (refers to 'dot line'). This phenomenon was also confirmed in the water content, $T_{\text {local }}$ and $R H_{\text {local }}$ profiles in Figs $11 \mathrm{a}, 11 \mathrm{~b}$ and $11 \mathrm{c}$ respectively (refers to 'dot line'). Both samples have different patterns due to the lowest value of $T_{\text {local }}$ and $R H_{\text {local }}$ relatively than other samples. Sample ' $\mathrm{C}$ ' have the range of $17.21{ }^{\circ} \mathrm{C} \pm 0.53 \%$ and $78.37 \% \pm 2.87$ while ' $\mathrm{D}$ ' have $16.90^{\circ} \mathrm{C} \pm 0.46$ and $79.33 \% \pm 2.61$. It can be concluded that the textural features can detect the different environmental condition in a plant factory. The variation of textural appearance influences moss affectivity in the function of water proofing, easy maintenance and comfortable greening technology.

The monitoring was stopped on day 17 , because the water content has already shown no decreasing effect of its subjected to drying conditions as shown in Fig. 12. Four quality statuses were identified to control moss affectivity (refers to 'circle sign'). Day 1 is soak appearance which is not affective to satisfy the consumer due to overwatering. Day 4 indicated the wet appearance which is affective to the consumer. Day 9 indicated the semi-dry appearance which is affective to the consumer. Finally, day 10 is dry appearance which is not affective to satisfy the consumer due to change of canopy size. The smaller size of canopy was reflected by the low value of water content as indicated in Fig. 12.

\section{Artificial neural network}

The experimental and questionnaire results are recapitulated in to 145 set data. The 133 set data were used in training ANN model, while the remains were used in inspection data. Table 4 indicated examples of three set training data and Table 5 for the output.

Based on sensitivity analysis of output error by trial and error basis (Table 6), eleven neurons in the hidden layer were determined. The architecture of network consisted of nine neurons in the input layer, eleven neurons in the hidden layer and three neurons in the output layer (9-11-3).

The training converged after approximately 40,000 iterations, learning coefficient of 0.1 and momentum 0.9. The mean value of Root Mean Square Error (RMSE) of training and inspection data was 0.020 and 0.025 respectively.

Comparison of training and inspection data in watchdog model was made in Figs. 13, 14 and 15 to show the closeness between predicted values by ANN and measured experimental values.

The values for coefficient of determination $\left(R^{2}\right)$ were predicted from 0.73 to 0.99 as shown in Table 7.

In addition, the examples of inspection data for different quality status were also performed in Fig.16 in order to indicate the possibility to use trained ANN as an intelligent watchdog model. By using these link weights from trained ANN, affectivity status, $T_{\text {local }}$ and $R H_{\text {local }}$ are estimated.

The low $R^{2}$ value on $R H_{\text {local }}$ in Table 7 is caused by the variation of $R H_{\text {setpoint }}$ training data (73 to $93.7 \%$ ). This range set point generated wide range of $R H_{\text {local }}$ as indicated in Fig. 15a (70 to $85 \%$ ) influences significantly the affectivity of moss material. The further experiment based on the shorter range of global relative humidity is suggested to increase the precision of watchdog model.

3. Research limitations and advantages

The limitation of the current research is samples which are generated. The water content which was reflected in the samples image was considered static. However, this problem could be overcome by converting the data base of samples $(8 \mathrm{~cm} \times 11 \mathrm{~cm})$ to the dynamic moss module $(50$ $\mathrm{cm} \times 50 \mathrm{~cm}$ ) for the actual application of greening technology.

The present research result is applicable for the irrigation management in greening technology. The objective is to satisfy the consumer in the functions of waterproofing, easy maintenance and comfortable. Water content and textural features of samples are applicable for detecting and controlling the pattern appearance of the module in the irrigation process. 
AN INTELLIGENT WATCHDOG MODEL

Table 4 Three examples of set input training data.

\begin{tabular}{clccc}
\hline No & Inputs & Set 1 & Set 2 & Set 3 \\
\hline 1 & Energy $\left(\times 10^{-4}\right)$ & 0.032 & 0.021 & 0.00016 \\
2 & LH $\left(\times 10^{-1}\right)$ & 0.84 & 0.67 & 0.044 \\
3 & Contrast & 481.47 & 559.81 & 1037.79 \\
4 & $T_{\text {set point }}$ & 19.7 & 15.6 & 19.4 \\
5 & $R H_{\text {set point }}$ & 82 & 91.0 & 81.7 \\
6 & Water content & 8.82 & 3.97 & 1.47 \\
7 & WAII $_{\text {waterprofing }}$ & 4.25 & 4.20 & 0.00 \\
8 & WAII $_{\text {easy maintenance }}$ & 4.45 & 4.43 & 0.00 \\
9 & WAII $_{\text {comfortable }}$ & 4.37 & 4.29 & 0.00 \\
\hline
\end{tabular}

Table 5 Three examples of output set training data.

\begin{tabular}{clccc}
\hline No & Outputs & Set 1 & Set 2 & Set 3 \\
\hline 1 & Affectivity status & 1 & 0.5 & 0 \\
2 & $T_{\text {local }}$ & 19.67 & 16.97 & 21.17 \\
3 & $R H_{\text {local }}$ & 75.00 & 79.67 & 67.33 \\
\hline
\end{tabular}

Table 6 Sensitivity analysis for intelligent watchdog model.

\begin{tabular}{ccccc}
\hline Learning coefficient & Momentum & Hidden nodes & Learning iteration & Mean RMSE \\
\hline 0.1 & 0.9 & 9 & 20,000 & 0.023 \\
0.1 & 0.9 & 11 & 10,000 & 0.024 \\
0.1 & 0.9 & 11 & 20,000 & 0.022 \\
0.1 & 0.9 & 11 & 40,000 & $0.020^{\mathrm{a}}$ \\
0.1 & 0.9 & 13 & 20,000 & 0.023 \\
\hline
\end{tabular}

a) minimum RMSE

Table $7 \quad R^{2}$ value of watchdog model.

\begin{tabular}{cccc}
\hline$R^{2}$ value & Affectivity status & $T_{\text {local }}$ & $R H_{\text {local }}$ \\
\hline Training & 0.99 & 0.92 & 0.76 \\
Inspection & 0.99 & 0.90 & 0.73 \\
\hline
\end{tabular}

\section{CONCLUSIONS}

The research results in a case study of moss greening (Rhacomitrium japonicum) concluded the possibility to control the affectivity of a bio-greening material using Artificial Neural Network (ANN). An intelligent watchdog model were tested successfully described the relationship between non-uniform controlled environment and material affectivity using back-propagation supervised learning method and inspection data. The trained ANN model generated satisfied correlation between predicted and measured values and minimum inspection error. It is suggested that the affectivity can be controlled by appropriate selection of ANN architecture.

On other side, fusing various parameters of bio-material, non-uniform controlled environment and consumer information will become an attractive input for developing an intelligent watchdog model in a plant factory. ANN is capable to predict affectivity status, local temperature and relative humidity. Affectivity status is possible as threshold quality status for the quality control. Local temperature and relative humidity is possible as the feedbacks for non-uniform environmental control system.

Finally the research results concluded that the intelligent watchdog model is applicable as a 


\section{USHADA AND H. MURASE}

new methodology for quality control in producing an affective bio-greening material. A plant factory can offer a set of reference samples appearance with a textural feature and water content database. The consumer evaluates easily the affectivity of a moss using its appearance. This appearance is detected in production using textural features extracted from grey level cooccurrences texture analysis and water content. Questionnaire concluded a set of design attributes which contributes significantly to material affectivity. Based on these design attributes and the data base, the material is produced in the non-uniform environment. Finally, it is controlled using ANN.

Mirwan would like to thank the Hitachi Scholarship Foundation Japan for funding his doctoral course in Osaka Prefecture University, and on the same line Gadjah Mada University Indonesia, Faculty of Agricultural Technology, and Department of Agro-industrial Engineering for giving a chance to continue study in Osaka Prefecture University, Japan.

\section{REFERENCES}

DBK. 2004. Pop Imaging Ver. 3.41, An Image Processing and Analysis. DBKids Inc. Japan.

Haralick, R. M., Dinstein, I., Shanmugam, K. 1973. Textural features for image classification, IEEE Trans. on Syst., Man, and Cybernetics. SMC 3: 610-621.

Hori, S., Taki, H., Washio, T., Motoda, H. 2002. Applying data mining to a field quality watchdog task. Electric. Eng. Jpn. 140: 18-25.

MI. 1998. Microsoft Visual C++ Ver.6.0. In Microsoft Visual Studio Net. Microsoft Inc., Japan.

Murase, H., Tani, A., Nishiura, Y., Kiyota, M. 1997. Growth monitoring of green vegetables cultured in a centrifuge phytotron. In "Plant Production in Closed Ecosystems" (ed. by Goto, E., Kurata, K., Hayashi, M., Sase, S.), Kluwer Academic Publishers, Dordrecht Netherlands, p 305-319.

Murase, H., Ushada, M. 2006. Machine vision applications for micro-precision agriculture. Environ. Control Biol. 44: 199-206.

Nagamachi, M. 1995. Kansei engineering: a new ergonomic consumer-oriented technology for product development. Int. J. Ind. Ergon. 15: 3-11.

NIH. 2004. Image-J, A Public Domain Java Image Processing Program. National Institutes of Health U.S.

Reed, R. D., Marks II, R. J. 1999. Neural smithing, supervised learning in feed forward artificial neural networks. Massachusetts Institute of Technology Press, p 1-6.

Takahashi, N., Murase, H., Murakami, K. 2002. Local temperature control within a confined space by using a neural network model. J. Soc. High Tech. Agric. 14: 131-135.

Ushada, M., Murase, H., Fukuda, H. 2007. Non-destructive sensing and its inverse model for canopy parameters using texture analysis and artificial neural network. Comput. Electron. Agric. 57: 149-165.

Yang, S., Nagamachi, M., Lee, S. 1999. Rule-based inference model for the Kansei engineering. Int. J. Ind. Ergon. 24: 459-471.

Zaidi, M. A., Murase, H., Honami, N. 1999. Neural network model for the evaluation of lettuce plant growth. J. Agric. Eng. Res. 74: 237-242. 